Research Paper

\title{
Non-Pegylated Liposomal Doxorubicin-Cyclopho- sphamide in Sequential Regimens with Taxanes as Neoadjuvant Chemotherapy in Breast Cancer Patients
}

\author{
Patrizia Vici ${ }^{\bowtie}$, Laura Pizzuti ${ }^{1}$, Teresa Gamucci², Domenico Sergi ${ }^{1}$, Francesca Conti ${ }^{1}$, Germano Zampa ${ }^{3}$, \\ Pietro Del Medico ${ }^{4}$, Roy De Vita ${ }^{5}$, Marcello Pozzi ${ }^{5}$, Claudio Botti ${ }^{6}$, Simona Di Filippo7 , Federica Tomao ${ }^{8}$, \\ Isabella Sperduti ${ }^{9}$ and Luigi Di Lauro ${ }^{1}$ \\ 1. Department of Medical Oncology B, Regina Elena National Cancer Institute, Via Elio Chianesi 53, 00144, Rome, Italy, Rome, Italy \\ 2. Medical Oncology Unit ASL Frosinone, Via Armando Fabi, 03100, Frosinone, Italy \\ 3. Oncology Unit, Nuovo Regina Margherita Hospital, Via Emilio Morosini 30, 00153, Rome, Italy \\ 4. Department of Medical Oncology, Reggio Calabria General Hospital, via Melacrino, 89100 Reggio Calabria, Italy \\ 5. Department of Plastic and Reconstructive Surgery, Regina Elena National Cancer Institute, Via Elio Chianesi 53, 00144, Rome, Italy \\ 6. Department of Breast Surgery, Regina Elena National Cancer Institute, Via Elio Chianesi 53, 00144, Rome, Italy \\ 7. Department of Hepatobiliary Surgery, Regina Elena National Cancer Institute, Via Elio Chianesi 53, 00144, Rome, Italy \\ 8. Department of Gynecologic and Obstetric Sciences, La Sapienza University of Rome, Viale Regina Elena 324, 00161, Rome, Italy \\ 9. Biostatistics Unit, Regina Elena National Cancer Institute, Via Elio Chianesi 53, 00144, Rome, Italy
}

$\triangle$ Corresponding author: Patrizia Vici, MD. Medical Oncology B, Regina Elena National Cancer Institute Via Elio Chianesi, 53, 00144 Rome (Italy). E-mail: pvici@ifo.it; Tel. 00390652665584; Fax: 00390652665075

(c) Ivyspring International Publisher. This is an open-access article distributed under the terms of the Creative Commons License (http://creativecommons.org/ licenses/by-nc-nd/3.0/). Reproduction is permitted for personal, noncommercial use, provided that the article is in whole, unmodified, and properly cited.

Received: 2014.03.17; Accepted: 2014.04.04; Published: 2014.04.25

\begin{abstract}
Purpose: Chemotherapy regimens containing anthracyclines and taxanes represent the landmark of neoadjuvant systemic therapy of breast cancer. In advanced breast cancer patients liposomal anthracyclines (LA) have shown similar efficacy and less cardiac toxicity when compared to conventional anthracyclines. We performed this retrospective analysis in order to evaluate the efficacy and tolerability of neoadjuvant regimens including LA outside of clinical trials in routine clinical practice.
\end{abstract}

Methods: Fifty operable or locally advanced, HER2 negative, breast cancer patients were retrospectively identified in 5 Italian cancer centres. Nineteen patients had received 4 cycles of non-pegylated liposomal doxorubicin (NPLD) and cyclophosphamide, followed by 4 cycles of docetaxel, every 3 weeks. In 25 patients the reverse sequence was employed, and a third subgroup of 6 patients received 4 cycles of NPLD/cyclophosphamide every 3 weeks followed by 4 cycles of weekly carboplatin and paclitaxel.

Results: We observed 10 pathological complete responses (PCR) $(20.0 \%, 95 \% \mathrm{Cl}, 9 \%$ to $31 \%)$, and $35(70 \%, 95 \% \mathrm{Cl}, 57.3 \%$ to $82.7 \%)$ partial responses (PPR), whereas no patients progressed onto therapy. In the small subset of triple negative tumors the PCR rate was $37.5 \%$, and in tumors expressing ER and/or PgR it was $16.7 \%$. A pCR rate of $26.5 \%$ was observed in tumors with high $\mathrm{Ki}-67$, whereas in tumors with low $\mathrm{Ki}-67$ only one $(6.2 \%) \mathrm{pCR}$ was observed $(p=0.14)$. Treatments were well tolerated. The most common toxicities were myelosuppression and palmar-plantar erytrodysesthesia; 4 asymptomatic and transient LVEF decrease have been recorded, without any case of clinical cardiotoxicity.

Conclusions: NPLD-cyclophosphamide and taxanes sequential regimens were proven effective and well tolerated in breast cancer patients with contra-indication to conventional anthracyclines undergoing neoadjuvant chemotherapy, even outside of clinical trials in everyday clinical practice.

Key words: Breast cancer, neoadjuvant chemotherapy, non-pegylated liposomal doxorubicin, retrospective analysis, everyday clinical practice 


\section{Background}

Neoadjuvant chemotherapy is commonly employed in operable and locally advanced breast carcinoma. It has several advantages, including downstaging the primary tumor with subsequent higher rates of breast conserving surgery, providing an in vivo chemosensibility test. Moreover, pathological complete response (pCR) at surgery is considered a surrogate marker for long-term outcome, even outside of clinical trials $(1,2)$. Current neoadjuvant treatments sequentially combine an anthracycline-based regimen followed or preceeded by a taxane. Although an optimal regimen has not yet been established, a combination of four cycles of an anthracycline-based regimen and four cycles of a taxane might produce the higher $\mathrm{pCR}$ rate which, in human epidermal growth factor receptor 2 (HER2) negative tumors, usually ranges from $22 \%$ to $31 \%(3,4)$. In regards to the sequential order of administering anthracycline and taxane, no definite data are reported (5), even if some experiences in the advanced and adjuvant setting indicate the reverse sequence, with the taxane administered first, as equally effective and possibly less toxic (6-9).

Among the most life-threatening side effects of anthracyclines is cardiac toxicity. It has been described since the 1970s, as mainly chronic or late-onset cardiotoxicity, frequently leading to congestive heart failure, and it is well known how the risk of cardiotoxicity increases with higher cumulative doses of anthracyclines $(10,11)$. The incidence and severity of cardiotoxicity is higher when anthracyclines are administered in bolus compared to continuous regimens, possibly related to higher plasmatic peak reached (12). Some predisponing factors are described, such as hypertension, age older than 65 years, previous mediastinal radiotherapy, concomitant use of other drugs such as paclitaxel, cyclophosphamide, trastuzumab. Guidelines recommend a maximum cumulative doxorubicin dose of $400-450 \mathrm{mg} / \mathrm{m}^{2}$, with reported $3-4 \%$ of clinical cardiotoxicity, being the incidence of congestive heart failure up to $18 \%$ in patients receiving $700 \mathrm{mg} / \mathrm{m}^{2}$ of doxorubicin. The incidence of congestive heart failure with epirubicin is lower, occurring in $0.7 \%$ of patients receiving a cumulative dose of $660 \mathrm{mg} / \mathrm{m}^{2}$, and cumulative doses for epirubicin should not exceed $800-900 \mathrm{mg} / \mathrm{m}^{2}$ $(11,13,14)$. Numerous trials evaluated different techniques aimed to an early diagnosis of anthracyclines cardiomyopathy, or the use of cardioprotectant agents (15-19), but the issue of anthracycline cardiotoxicity still remains a significant challenge, particularly in the neoadjuvant and adjuvant breast cancer settings, where the goal of treatment is cure.

Encapsulating anthracyclines into liposomes al- low patients to receive much higher doses of anthracycline delivered mainly into the tumor, while maintaining activity and fewer side effects, and formulations for clinical use are now available in clinical practice, slightly differing in pharmacological properties (20). The two formulations employed in breast cancer are the pegylated form (Caelix/Doxil $\left.{ }^{\circledR}\right)$, and the non-pegylated doxorubin (NPLD, Myocet $\left.{ }^{\circledR}\right)$. The latter was approved for the treatment of advanced breast cancer, and showed efficacy as a single agent or in combination with other drugs both in anthracycline-naïve and in pretreated breast cancer patients $(21,22)$.

Liposomal anthracyclines (LAs) have shown equal efficacy in phase III trials, when compared with conventional anthracyclines in advanced breast cancer patients. Their unique formulation offers the advantages of lower specific toxicities and possibly increase efficacy, achieving a higher therapeutic index (23-28). The advantages associated to the use of these drugs opens a remarkable window of opportunity within a wider range of breast cancer patients, across different settings and including unfit or frail patients.

Data concerning cardiotoxicity were particularly encouraging, with a lower incidence of asymptomatic and symptomatic cardiac toxicity. In 2010, a systematic review on cardiotoxicity of different anthracycline compounds, confirmed that LAs strongly reduced the overall risk of cardiotoxicity $(R R=0.38, p<0.0001)$ and the risk of clinical heart failure $(R R=0.20, p=0.02)$ (29).

A recent meta-analysis, including nine randomized trials comparing in various tumors including advanced breast cancer, and liposomal and conventional anthracyclines, confirms that liposomal formulation offers an alternative to conventional anthracyclines for patients with previous history of cardiac disease, elderly or "frail". Moreover, the incidence of other toxicities, such as haematological toxicity, were lower than those with conventional anthracyclines, making the liposomal formulation more attractive for elderly patients (30). Recently, the results of the CASA randomized adjuvant trial, evaluating pegylated liposomal doxorubicin and low dose metronomic cyclophosphamide and methotrexate in elderly and "frail" patients with triple negative advanced breast cancer have been published reporting favourable results (31).

In fact, the use of conventional anthracyclines in the neoadjuvant setting may warrant some level of risk, particularly in "frail" patients, such as patients with cardiovascular risks, or previous cardiac disease, or elderly patients with co-morbidities contra-indicating the use of "standard chemotherapy regimens". 
A number of small clinical trials with LAs in the neoadjuvant setting have been published, where LAs were combined with docetaxel and gemcitabine, or with paclitaxel, all reporting satisfactory activity and absence of cardiotoxicity $(32,33)$, even if most of them are in HER2 positive tumors, in combination with trastuzumab $(34,35)$.

In order to evaluate efficacy and tolerability of neoadjuvant regimens including LAs in "real-world" population, we performed a retrospective analysis of the results of NPLD-containing regimens followed or preceded by taxanes as neoadjuvant treatment in operable or locally advanced, HER2 negative, breast cancer patients with contra-indication to conventional anthracyclines.

\section{Patients and methods}

Patient clinical information, tumor characteristics, response rate and toxicity information were recorded. From April 2007 to February 2013, a total of 50 patients with operable or locally advanced breast cancer were retrospectively identified in 5 Italian cancer centres.

All primary breast cancers had undergone a core biopsy prior to neoadjuvant treatment, and staging work-up included complete blood count, chemistry, chest radiography, liver ultrasound or computed tomography scan of the liver and bone scan. Cardiac function evaluation included clinical history, a baseline left ventricular ejection fraction (LVEF) evaluation by echocardiogram, and an electrocardiogram, all repeated after 4 cycles, at the end of neoadjuvant chemotherapy, and during the follow up period, every 6 months or whenever indicated. All the evaluated patients had normal organ functions, aged $>18$ years, and ECOG performance status (PS) $\leq 2$. However, these patients were not considered suitable for "standard chemotherapy regimens", due to co-morbidities, age (30 out of 50 patients were $\geq 65$ years old), or "frailty".

The assessment of proliferative index [percentage of Ki-67 stained cells], estrogen receptor (ER), progesterone receptor (PgR) and HER2 were determined by standard methods on pre-treatment core-biopsy, and evaluation was repeated, whenever feasible, at definite surgery. Immunoreactions were revealed by a streptavidin-biotin enhanced immunoperoxidase technique in an automated autostainer (Bond ${ }^{\mathrm{TM}}$ Max, Menarini, Florence, Italy). ER and PgR expression were tested using mAb 6F11 (Menarini) and mAb 1A6 (Menarini) respectively, proliferative index using the anti $\mathrm{Ki}-67 \mathrm{mAb}$ (MIB1, Dako), for HER2 overexpression using the polyclonal antibody A0485 (Dako). ER and PgR were considered positive when $>1 \%$ of the neoplastic cells showed distinct nu- clear immunoreactivity, whereas Ki-67, based on the median value of our series, was regarded as high if more than $14 \%$ of the cell nuclei were immunostained. Patients with HER2 positive tumors at baseline (Dako $3+$, or $2+$ but amplified by FISH or SISH) were excluded from the present analysis.

The choice of administering NPLD instead of a conventional anthracycline was determined by one or more of the following reasons: low PS, age $\geq 70$ years, previous cardiac disease, severe or uncontrolled hypertension, "borderline" baseline LVEF ( $45 \%$ to $49 \%$ ), or other medical conditions contra-indicating the use of "conventional" anthracycline-based regimens.

After standard premedication, 19 patients had received 4 cycles of NPLD $\left(60 \mathrm{mg} / \mathrm{m}^{2}\right)$ in combination with cyclophosphamide $\left(600 \mathrm{mg} / \mathrm{m}^{2}\right)$, followed by 4 cycles of docetaxel $\left(100 \mathrm{mg} / \mathrm{m}^{2}\right)$, with a three-weekly schedule. The reverse sequence was employed in 25 patients with docetaxel given prior to NPLD/cyclophosphamide regimen. A third subgroup of 6 patients received 4 cycles of NPLD/cyclophosphamide every 3 weeks followed by a weekly administration of carboplatin (AUC 2.5) and paclitaxel $\left(80 \mathrm{mg} / \mathrm{m}^{2}\right)$, on days $1,8,15$ every 4 weeks for 4 cycles. Prophylactic granulocyte colony stimulating factors (G-CSF) were administered in the majority of patients, and all the patients received G-CSF in case of severe myelosuppression.

Toxicity was assessed at each treatment cycle using the National Cancer Institute Common Terminology Criteria (version 4.0).

Pathological response was evaluated in all enrolled patients at definite surgery. Pathological complete response ( $\mathrm{pCR}$ ) was defined as no residual invasive tumor in both breast and axilla (1).

Surgery procedures included breast conserving surgery or mastectomy, and complete axillary or sentinel node dissection. After surgery, patients were treated with standard radiotherapy and hormonal adjuvant therapy, whenever indicated.

\section{Statistical design}

Patient and disease characteristics were analysed using descriptive statistics, and expressed as either relative frequency [percentages] for discrete variables or median for continuous variables. The association between variables was tested by the Pearson Chi-Square or Fisher Exact test, when appropriate. Relapse-free survival (RFS) were calculated from date from definite surgery to local or distant invasive relapse, death, or last follow-up and plotted as Kaplan-Meier curves. The SPSS (21.0) statistical program was used for all analyses.

Percentages of $\mathrm{pCR}$ were reported with their exact binomial 95\% confidence intervals (CIs). 
This retrospective analysis was approved by the coordinating centre's Ethics Committee at the Regina Elena National Cancer Institute, Rome, and was carried out according the principles of the Declaration of Helsinki.

\section{Results}

\section{Patients characteristics}

From April 2007 to February 2013, 50 patients were retrospectively identified from 5 Italian cancer centres. Main patient characteristics are listed in Table 1. Median age was 67 (range, 34-76), with 30 patients older than 65 years; median PS status was 1 (range, $0-2)$; 42 tumors (84\%) were ER and or PgR positive, 8 tumors were triple negative (TN). Clinical stage at baseline ranged from IIA to IIIC. The baseline mean breast tumor size was $4.5 \mathrm{~cm} ; 48 \%$ of the patients had stage III disease, and 2 patients (4\%) had inflammatory breast cancer. Forty-four tumors were invasive ductal carcinoma, and 6 tumors were invasive lobular carcinoma. Neoadjuvant treatment regimens are listed in Table 2.

Table I. Main baseline patient characteristics in 50 patients

\begin{tabular}{ll}
\hline Characteristics & N (\%) \\
\hline Age & \\
Median & 67 \\
Range & $34-76$ \\
Menopausal status & \\
Pre & $20(40)$ \\
Post & $30(60)$ \\
Histology & \\
Ductal & $44(88)$ \\
Lobular & $6(12)$ \\
Tumor size & \\
T1 & $6(12)$ \\
T2 & $20(40)$ \\
T3 & $12(24)$ \\
T4 & $12(24)$ \\
Clinical stage & \\
IIA & $10(20)$ \\
IIB & $16(32)$ \\
IIIA & $9(18)$ \\
IIIB & $13(26)$ \\
IIIC & $2(4)$ \\
G1 & \\
G2 & \\
G3 & $5(10)$ \\
Unknown & $10(20)$ \\
$\leq 14 \%$ & $24(48)$ \\
$>14 \%$ & $11(22)$ \\
Hormone receptor status & \\
Positive & $16(32)$ \\
Negative & $34(68)$ \\
\hline & $42(84)$ \\
Ming & $8(16)$ \\
\hline
\end{tabular}

Table 2. Neoadjuvant chemotherapy regimens in 50 patients

\begin{tabular}{ll}
\hline Neoadjuvant chemotherapy regimens & $\begin{array}{l}\text { Patients } \\
{[\%]}\end{array}$ \\
\hline $\begin{array}{l}\text { NPLD/cyclophosphamide (4 cycles) } \rightarrow \text { docetaxel (4 cy- } \\
\text { cles) }\end{array}$ & $19(38)$ \\
$\begin{array}{l}\text { Docetaxel (4 cycles) } \rightarrow \text { NPLD/cyclophosphamide (4 } \\
\text { cycles) }\end{array}$ & $25(50)$ \\
$\begin{array}{l}\text { NPLD/cyclophosphamide (4 cycles) } \rightarrow \text { weekly car- } \\
\text { boplatin and paclitaxel (4 cycles) }\end{array}$ & $6(12)$ \\
\hline NPLD: Non-pegylated liposomal doxorubicin. &
\end{tabular}

\section{Efficacy}

Forty-five patients had an objective clinical response to neoadjuvant treatment, 5 patients had a stable disease. A pCR at surgery was observed in 10 patients $(20.0 \%, 95 \% \mathrm{CI}, 9 \%$ to $31 \%)$; in 35 patients a partial response (pPR) was observed $(70 \%, 95 \% \mathrm{CI}$, $57.3 \%$ to $82.7 \%$ ), whereas no patients progressed onto therapy (Table 3). In 1 patient achieving a pCR, isolated tumor cells were observed in the breast at surgery.

Table 3. Pathological responses in 50 patients

\begin{tabular}{lll}
\hline Responses & $\begin{array}{l}\text { No. of pa- } \\
\text { tients }\end{array}$ & \% (C.I. 95\%) \\
\hline Complete response* & 10 & $20.0(9-31)$ \\
Partial response & 35 & $70(57.3-82.7)$ \\
Stable disease & 5 & 10 \\
\hline * Pathological complete response (pCR) was defined as no residual invasive tumor
\end{tabular}

in both breast and axilla (1)

Among the small subset (8 patients) with $\mathrm{TN}$ tumors, we observed 3 pCR $(37.5 \%)$, whereas in tumors expressing ER and or PgR the pCR rate was $16.7 \%$. A pCR rate of $26.5 \%$ was observed in 34 tumors with high (>14\%) Ki-67; conversely, in 16 tumors with low $(<14 \%) \mathrm{Ki}-67$, only one $(6.2 \%)$ pCR was observed $(p=0.14)$. In the two cases of inflammatory breast cancers, one $\mathrm{PCR}$ and one PPR were observed.

In regards to the sequential order of administering LAs and taxanes, no apparent differences were observed in treatment efficacy or toxicity between the two schedules, but sample size is small.

In regards to surgery procedures, 30 patients $(60.0 \%)$ underwent mastectomies, whereas $20(40.0 \%)$ patients had breast conserving surgery. A complete axillary dissection was performed in 44 patients $(88 \%)$, whereas a sentinel node biopsy was performed in 6 patients $(12 \%)$. Forty-two patients with tumor expressing hormonal receptors at baseline biopsy received adjuvant hormonal therapy for 5 years; postoperative radiotherapy was administered as clinically indicated. In 1 patient with residual tumor at surgery, HER2 was negative at baseline biopsy, but resulted 
amplified at definite surgery, and the patient was treated with adjuvant trastuzumab for 1 year.

With a median follow up of 48 months (range, 6 to 64 months), 5 recurrences were observed: 2 lymphonodes and skin, 3 visceral, and 1 second primary lung cancer was recorded. As expected, no recurrences were observed in the 10 patients who achieved a pCR, disease progression being observed only in patients not achieving a $\mathrm{pCR}$. The 3-years relapse-free survival was $87.0 \%$ (Figure 1 ).

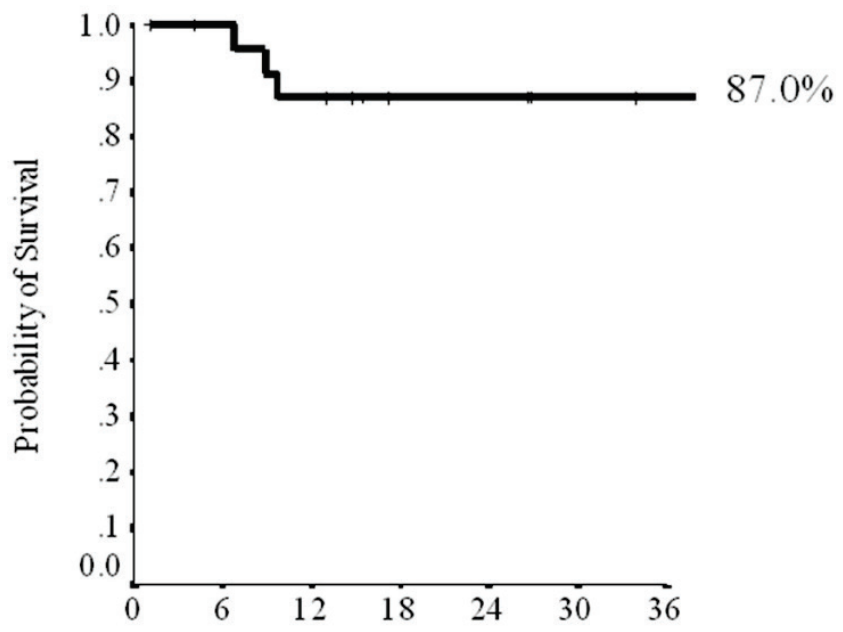

Months

Figure I. Three-years relapse free survival.

\section{Toxicity}

Regarding treatment tolerability, regimens were quite well tolerated overall. Forty-four patients (92\%) completed eight cycles of neoadjuvant treatment, with 2 patients discontinuing docetaxel regimen after 2 and 3 cycles, (at the $6^{\text {th }}$ and $7^{\text {th }}$ cycle of the whole chemotherapy plan), respectively, for G4 neutropenia, and 2 patients discontinuing NPLD after the third cycle of the second part of chemotherapy plan for G3 palmo-plantar erytrodysesthesia (PPE).

The safety of the regimens is reported in Table 4 . The main toxicity observed during treatment administration was myelosuppression, occurring mostly while on docetaxel administration, with grade 3-4 neutropenia observed in 22 patients $(24 \%$ and $20 \%$, respectively), and PPE, which was of grade 3 in 4 patients $(8 \%)$; neutropenic fever was observed in 3 patients $(6 \%)$, during docetaxel-based regimen. G-CSF had been administered in all but 8 patients (prophylactively in 33 and after significant neutropenia in 9 patients). No episodes of grade 3 or 4 vomiting was observed. Mucositis was common, but usually mild, being of grade 3 in 2 patients (4\%), and occurring on docetaxel administration. Severe alopecia was uncommon during NPLD administration, in contrast it was universal while on taxanes administration. No severe neurotoxicity was observed, being only of grade 1-2. An allergic reaction to docetaxel, consisting in flushing and pruritus, was observed in 9 patients, not requiring treatment discontinuation. A 25\% docetaxel dose-reduction was performed in 5 patients, due to myelosuppression (4 patients) or mucositis (1 patient). As reported above, in 2 patients NPLD had to be discontinued after the third cycle (after 7 cycles of chemotherapy plan) due to grade 3 PPE. As to cardiotoxicity, treatments were very well tolerated, with no cases of clinical cardiotoxicity. All the patients had at least a baseline echocardiogram and 2 subsequent evaluations after 4 and 8 cycles of neoadjuvant program, and were included in the cardiac function analysis. The median cardiac follow-up period was 48 months (range, 6- 64 months). We recorded only 4 cases $(8 \%)$ of asymptomatic LVEF decrease (ranging from $10 \%$ to $15 \%$ ), all occurring at the end of the NPLD administration, with a prompt recovery within 3 months. No differences in mean LVEF values according to NPLD regimen were recorded.

Overall, no significant differences in toxicity were observed according to age or any other prognostic factor. No toxic deaths were observed.

Table 4. Main toxicities in 50 patients according to National Cancer Institute Common Terminology Criteria version 4.0

\begin{tabular}{|c|c|c|c|c|}
\hline Toxicity & $\begin{array}{l}\text { Grade } 1 \\
\%\end{array}$ & $\begin{array}{l}\text { Grade } 2 \\
\%\end{array}$ & $\begin{array}{l}\text { Grade } 3 \\
\%\end{array}$ & $\begin{array}{l}\text { Grade } 4 \\
\%\end{array}$ \\
\hline \multicolumn{5}{|l|}{ Hematologic } \\
\hline Leukopenia & 44 & 22.2 & 15.5 & 2.2 \\
\hline Neutropenia $^{a}$ & 28 & 22.2 & 24.4 & 20 \\
\hline Thrombocytopenia & 18 & 8.9 & - & - \\
\hline Anemia & 18 & 17.8 & 4.5 & - \\
\hline \multicolumn{5}{|l|}{ Nonhemathologic } \\
\hline PPE b & 44 & 40 & 8 & \\
\hline Nausea/Vomiting & 22 & 20 & - & - \\
\hline Mucositis & 28 & 14 & 4 & - \\
\hline Diarrhea & 14 & - & - & - \\
\hline Fatigue & 54 & 20 & - & - \\
\hline Neurotoxicity & 40 & 6 & - & - \\
\hline Hypertransaminases & 18 & 14 & 2 & - \\
\hline Hypersensivity & 8 & 10 & - & - \\
\hline Cardiac toxicity & 8 & - & - & - \\
\hline
\end{tabular}

aFebrile neutropenia in 3 patients $(6 \%)$. ${ }^{\mathrm{b}}$ PPE: Palmar-plantar erytrodysesthesia

\section{Discussion}

Anthracyclines comprise of a group of the most active cytotoxic agents in breast cancer which are routinely administered in the pre-operative treatment for operable or locally advanced breast cancer. However, their limits reside in the low therapeutic index, 
mainly for cumulative cardiotoxicity, where current standard chemotherapy regimens may not be adequate for all breast cancer patients, such as elderly patients or patients with comorbidities. In recent years in fact, elderly women make up a large portion of breast cancer patients and, after a geriatric assessment, only a minor part are considered "fit", with the remaining portion showing one or more physical or functional limitations or familiar/social problems, thus categorized "frail". Moreover, there are patients that, even if young, have "borderline" medical conditions, such as unsatisfactory renal, liver function, or cardiac function, or poor performance status. In this setting, it may be more appropriate to substitute "classic" anthracyclines with LAs, which are known to concentrate into neoplastic tissue and to reduce the exposure of the drug to normal tissues, showing a favourable safety profile with comparable clinical activity $(26,27)$.

Neoadjuvant chemotherapy has been widely used in patients with operable and locally advanced breast cancer in order to increase the rate of breast conserving surgery and to obtain a better long-term outcome, and sequential regimens of anthracycline-based schemes followed or preceded by a taxane are considered the "standard" even outside clinical trials (36-39). Given the clinical relevance of including anthracyclines in the neoadjuvant setting (40), the use of a liposomal delivery system may improve the therapeutic ratio, as previously reported by neoadjuvant studies employing LAs in HER2 positive tumors in combination with trastuzumab (41).

The results of our study suggest that NPLD may offer an attractive alternative to conventional anthracyclines in the treatment of early breast cancer. In this retrospective multicentric analysis, we observed a pCR of $20 \%$, which is not dissimilar to the pCR rate observed with conventional anthracyclines and sequential taxane-based regimens in the subgroup of HER2 negative tumors $(3,4)$. In fact, in our case study more than $80 \%$ of the tumors were ER and/or PgR positive at baseline, and it is well known how this subset of tumors usually reaches low rates of $\mathrm{pCR}$ after neoadjuvant treatments (2). Moreover, a $40 \%$ of breast-conservative surgery was observed, even if $48 \%$ of the tumors were stage III at baseline. All the 3 treatment regimens employed were well tolerated, grade 3-4 toxicities were observed rarely, mostly during docetaxel delivery. The only grade 3 side-effect, possibly related to NPLD administration, was PPE, observed in 4 patients $(8 \%)$. This side effect was usually cumulative-dose related, occurring with NPLD doses higher than $180 \mathrm{mg} / \mathrm{m}^{2}$, and prompted treatment discontinuation in 2 patients after the third cycle. In the remaining 2 patients PPE was of grade 2, evolving to grade 3 only at the end of the neoadjuvant treatment, with subsequent rapid improvement and disappearance.

It is too early to evaluate whether achieving a pCR will result in predicting long-term favourable outcome, since the follow-up period is still too short, even if, to date, recurrences observed have only been in patients not experiencing a $\mathrm{pCR}$. We recognise that our study is limited by its retrospective design, lack of an appropropriate comparator group, limited follow-up period and small number of patients but, even though complete serial cardiac evaluation information was not available for all patients, the absence of any symptomatic cardiac toxicity, and the low incidence (8\%) of asymptomatic and transient LVEF decrease seem to confirm the cardiac safety of NPLD-based regimens as neoadjuvant treatment, even in "frail" patients, and in routine clinical practice.

The majority of the clinical trials employing LA in breast cancer are in advanced disease. The randomized trials of Batist and Harris first confirmed the equivalent activity and lower toxicity of NPLD in comparison with conventional doxorubicin $(25,26)$. The phase III randomized trial by Chan, comparing NPLD versus epirubicin, in combination with cyclophosphamide, as first-line chemotherapy, showed comparable efficacy and tolerability, even in terms of cardiotoxicity, even though epirubicin is known to be less cardiotoxic than doxorubicin (27). A more recent pilot randomized study evaluated a conventional chemotherapy regimen including epirubicin versus a NPLD-based regimen in advanced breast cancer, and the tissue doppler imaging (TDI) evaluation confirmed the low cardiotoxicity of liposomal doxorubi$\operatorname{cin}(42)$.

The biological heterogeneity of breast cancer is well known, and the identification of markers predictive of therapeutic response is a major challenge in breast cancer, mainly in the neoadjuvant setting, as well as incorporation of biological agents in primary treatment regimens. Significant encouraging results are achieved mainly in HER2 positive tumors, whereas in TN or in ER and/or PgR positive tumors established and efficacious targeted agents are lacking, being chemotherapy and endocrine therapy still the mainstay of treatment. In this setting, the availability of neoadjuvant sequential regimens devoid of significant cardiotoxicity and with a favourable therapeutic index, even in "frail" patients, may be of paramount relevance in routine clinical practice.

In conclusion, the results from this multicenter retrospective analysis on neoadjuvant NPLD-cyclophosphamide and taxanes sequential regimens in operable and locally advanced HER2 negative breast cancer patients with contra-indication 
to anthracyclines are encouraging, even with the limitation of the small sample size. Even if further data are required to confirm these promising findings, particularly in the longer term, our analysis indicate an efficacy comparable to the literature data, and confirmed a favourable toxicity profile, suggesting that NPLD-based regimens as primary chemotherapy may improve therapeutic index in patients not suitable to receiving a conventional anthracycline-based neoadjuvant chemotherapy, and outside of clinical trials in everyday clinical practice.

\section{Acknowledgements}

We thank Anna Maria Edlisca and Tania Merlino for technical assistance.

\section{Competing Interests}

The authors have declared that no competing interest exists.

\section{References}

1. Kuerer HM, Newman LA, Smith TL, et al. Clinical course of breast cancer patients with complete pathologic primary tumor and axillary lymph node response to doxorubicin-based neoadjuvant chemotherapy. J Clin Oncol. 1999; 17: 460-9.

2. Angelucci D, Tinari N, Grassadonia A, et al. Long-term outcome of neoadjuvant systemic therapy for locally advanced breast cancer in routine clinical practice. J Cancer Res Clin Oncol. 2013; 139: 269-80.

3. Bear HD, Anderson S, Brown A, et al. The effect on tumor response of adding sequential preoperative docetaxel to preoperative doxorubicin and cyclophosphamide: preliminary results from National Surgical Adjuvant Breast and Bowel Project Protocol B-27. J Clin Oncol. 2003; 21: 4165-74.

4. Sachelarie I, Grossbard ML, Chadha M, Feldman S, Ghesani M, Blum RH. Primary systemic therapy of breast cancer. Oncologist. 2006; 11: 574-89.

5. Vici P, Viola G, Rossi S, et al. Optimal sequence of anthracyclines and taxanes as adjuvant breast cancer treatment. Clin Ter. 2008; 159: 453-6.

6. Thiery-Vuillemin A, Llombart-Cussac A, Chaigneau L, et al. Sequential taxane and anthracycline-containing neoadjuvant regimens: the sequential order impact. Breast. 2011; 20: 46-9.

7. Vici P, Brandi M, Giotta F, et al. A multicenter phase III prospective randomized trial of high-dose epirubicin in combination with cyclophosphamide [EC] versus docetaxel followed by EC in node-positive breast cancer. GOIM [Gruppo Oncologico Italia Meridionale] 9902 study: Ann Oncol. 2012; 23: 1121-9.

8. Puhalla S, Mrozek E, Young D, et al. Randomized phase II adjuvant trial of dose-dense docetaxel before or after doxorubicin plus cyclophosphamide in axillary node-positive breast cancer. J Clin Oncol. 2008; 26: 1691-7.

9. Piedbois P, Serin D, Priou F, et al. Dose-dense adjuvant chemotherapy in node-positive breast cancer: docetaxel followed by epirubicin/cyclophosphamide $[\mathrm{T} / \mathrm{EC}]$, or the reverse sequence $[\mathrm{EC} / \mathrm{T}]$, every 2 weeks, versus docetaxel, epirubicin and cyclophosphamide [TEC] every 3 weeks. AERO B03 randomized phase II study. Ann Oncol. 2007; 18: 52-7.

10. Von Hoff DD, Layard MW, Basa P, et al. Risk factors for doxorubicin-induced congestive heart failure. Ann Intern Med. 1979; 91: 710-7.

11. Swain SM, Whaley FS, Ewer MS. Congestive heart failure in patients treated with doxorubicin: a retrospective analysis of three trials. Cancer. 2003; 97: 2869-79.

12. Lao J, Madani J, Puértolas T, et al. Liposomal doxorubicin in the treatment of breast cancer patients: a review. J Drug Deliv. 2013; 2013: 456409.

13. Fisher NG, Marshall AJ. Anthracycline-induced cardiomyopathy. Postgrad Med J. 1999; 75: 265-8.

14. Launchbury AP, Habboubi N. Epirubicin and doxorubicin: a comparison of their characteristics, therapeutic activity and toxicity. Cancer Treat Rev. 1993; 19: $197-228$.

15. Mladosievicova B, Urbanova D, Radvanska E, Slavkovsky P, Simkova I. Role of NT-proBNP in detection of myocardial damage in childhood leukemia survivors treated with and without anthracyclines. J Exp Clin Cancer Res. 2012; 31: 86.

16. Roziakova L, Bojtarova E, Mistrik M, et al. Serial measurements of cardiac biomarkers in patients after allogeneic hematopoietic stem cell transplantation. J Exp Clin Cancer Res. 2012; 31: 13.
17. Lopez M, Vici P, Di Lauro L, et al. Randomized prospective clinical trial of high-dose epirubicin and dexrazoxane in patients with advanced breast cancer and soft tissue sarcomas. J Clin Oncol. 1998; 16:86-92.

18. Swain SM, Vici P. The current and future role of dexrazoxane as a cardioprotectant in anthracycline treatment: expert panel review. J Cancer Res Clin Oncol. 2004; 130: 1-7.

19. Maini CL, Sciuto R, Ferraironi A, et al. Clinical relevance of radionuclide angiography and antimyosin immunoscintigraphy for risk assessment in epirubicin cardiotoxicity. J Nucl Cardiol. 1997; 4: 502-8.

20. Wang X, Yang L, Chen ZG, Shin DM. Application of nanotechnology in cancer therapy and imaging. CA Cancer J Clin. 2008; 58: 97-110.

21. Rosati MS, Raimondi C, Baciarello G, et al. Weekly combination of non-pegylated liposomal doxorubicin and taxane in first-line breast cancer: wALT trial [phase I-II]. Ann Oncol. 2011; 22: 315-20.

22. Curtit E, Nouyrigat P, Dohollou N, et al. Myotax: a phase II trial of docetaxel plus non-pegylated liposomal doxorubicin as first-line therapy of metastatic breast cancer previously treated with adjuvant anthracyclines. Eur J Cancer. 2011; 47: 2396-402.

23. Vici P, Colucci G, Giotta F, et al. A multicenter prospective phase II randomized trial of epirubicin/vinorelbine versus pegylated liposomal doxorubicin/vinorelbine as first-line treatment in advanced breast cancer. A GOIM study. J Exp Clin Cancer Res. 2011; 30: 39.

24. O'Brien ME, Wigler N, Inbar M, et al. Reduced cardiotoxicity and comparable efficacy in a phase III trial of pegylated liposomal doxorubicin $\mathrm{HCl}$ [CAELYX/Doxil] versus conventional doxorubicin for first-line treatment of metastatic breast cancer. Ann Oncol. 2004; 15: 440-9.

25. Harris L, Batist G, Belt R, et al. Liposome-encapsulated doxorubicin compared with conventional doxorubicin in a randomized multicenter trial as first-line therapy of metastatic breast carcinoma. Cancer. 2002; 94: 25-36.

26. Batist G, Ramakrishnan G, Rao CS, et al. Reduced cardiotoxicity and preserved antitumor efficacy of liposome-encapsulated doxorubicin and cyclophosphamide compared with conventional doxorubicin and cyclophosphamide in a randomized, multicenter trial of metastatic breast cancer. J Clin Oncol. 2001; 19: 1444-54.

27. Chan S, Davidson N, Juozaityte E, et al. Phase III trial of liposomal doxorubicin and cyclophosphamide compared with epirubicin and cyclophosphamide as first-line therapy for metastatic breast cancer. Ann Oncol. 2004; 15: 1527-34.

28. Sparano JA, Makhson AN, Semiglazov VF, et al. Pegylated liposomal doxorubicin plus docetaxel significantly improves time to progression without additive cardiotoxicity compared with docetaxel monotherapy in patients with advanced breast cancer previously treated with neoadjuvant-adjuvant anthracycline therapy: results from a randomized phase III study. J Clin Oncol. 2009; 27: 4522-9.

29. van Dalen EC, Michiels EM, Caron HN, Kremer LC. Different anthracycline derivates for reducing cardiotoxicity in cancer patients. Cochrane Database Syst Rev. 2010; 3: CD005006.

30. Rafiyath SM, Rasul M, Lee B, Wei G, Lamba G, Liu D. Comparison of safety and toxicity of liposomal doxorubicin vs. conventional anthracyclines: a meta-analysis. Exp Hematol Oncol. 2012; 1: 10.

31. Crivellari D, Gray KP, Dellapasqua S, et al. Adjuvant pegylated liposomal doxorubicin for older women with endocrine nonresponsive breast cancer who are NOT suitable for a "standard chemotherapy regimen": the CASA randomized trial. Breast. 2013; 22: 130-7.

32. Schmid P, Krocker J, Jehn C, et al. Primary chemotherapy with gemcitabine as prolonged infusion, non-pegylated liposomal doxorubicin and docetaxel in patients with early breast cancer: final results of a phase II trial. Ann Oncol. 2005; 16: 1624-31.

33. Gogas H, Papadimitriou C, Kalofonos HP, et al. Neoadjuvant chemotherapy with a combination of pegylated liposomal doxorubicin [Caelyx] and paclitaxel in locally advanced breast cancer: a phase II study by the Hellenic Cooperative Oncology Group. Ann Oncol. 2002; 13: 1737-42.

34. Antón A, Ruiz A, Seguí MA, et al. Phase I clinical trial of liposomal-encapsulated doxorubicin citrate and docetaxel, associated with trastuzumab, as neo-adjuvant treatment in stages II and IIIA, HER2-overexpressing breast cancer patients. GEICAM 2003-03 study. Ann Oncol. 2009; 20: 454-9.

35. Antón A, Ruiz A, Plazaola A, et al. Phase II clinical trial of liposomal-encapsulated doxorubicin citrate and docetaxel, associated with trastuzumab, as neoadjuvant treatment in stages II and IIIA HER2-overexpressing breast cancer patients. GEICAM 2003-03 study. Ann Oncol. 2011; 22: 74-9.

36. Smith IC, Heys SD, Hutcheon AW, et al. Neoadjuvant chemotherapy in breast cancer: significantly enhanced response with docetaxel. J Clin Oncol. 2002; 20: 1456-66.

37. von Minckwitz G, Raab G, Caputo A, et al. Doxorubicin with cyclophosphamide followed by docetaxel every 21 days compared with doxorubicin and docetaxel every 14 days as preoperative treatment in operable breast cancer: the GEPARDUO study of the German Breast Group. J Clin Oncol. 2005; 23: 2676-85.

38. Iwata H, Sato N, Masuda $\mathrm{N}$, et al. Docetaxel followed by fluorouracil/epirubicin/ cyclophosphamide as neoadjuvant chemotherapy for patients with primary breast cancer. Jpn J Clin Oncol. 2011; 41: 867-75.

39. Wu J, Li S, Jia W, Su F. Response and prognosis of taxanes and anthracyclines neoadjuvant chemotherapy in patients with triple-negative breast cancer. J Cancer Res Clin Oncol. 2011; 137: 1505-10. 
40. Kaufmann M, Hortobagyi GN, Goldhirsch A, et al. Recommendations from an international expert panel on the use of neoadjuvant [primary] systemic treatment of operable breast cancer: an update. J Clin Oncol. 2006; 24: 1940-9.

41. Saracchini S, Foltran L, Tuccia F, et al. Phase II study of liposome-encapsulated doxorubicin plus cyclophosphamide, followed by sequential trastuzumab plus docetaxel as primary systemic therapy for breast cancer patients with HER2 overexpression or amplification. Breast. 2013; 22: 1101-7.

42. Lotrionte M, Palazzoni G, Abbate A, et al. Cardiotoxicity of a non-pegylated liposomal doxorubicin-based regimen versus an epirubicin-based regimen for breast cancer: The LITE [Liposomal doxorubicin-Investigational chemotherapy-Tissue doppler imaging Evaluation] randomized pilot study. Int J Cardiol. 2013; 167: 1055-7. 